\title{
Hacking Health: Bottom-up Innovation for Healthcare Jeeshan Chowdhury
}

\author{
"The great driver of scientific innovation and technological" \\ innovation has been the historic increase in connectivity and \\ our ability to reach out and exchange ideas with other people. \\ And to borrow other people's hunches and combine them with \\ our hunches and turn them into something new. \\ Steve Johnson \\ Author of Where Good Ideas Come From: \\ The Natural History of Innovation
}

\begin{abstract}
Healthcare is not sustainable and still functions with outdated technology (e.g., pagers, paper records). Top-down approaches by governments and corporations have failed to deliver digital technologies to modernize healthcare. Disruptive innovation must come from the ground up by bridging the gap between front-line health experts and innovators in the latest web and mobile technology. Hacking Health is a hackathon that is focused on social innovation more than technical innovation. Our approach to improve healthcare is to pair technological innovators with healthcare experts to build realistic, human-centric solutions to front-line healthcare problems.
\end{abstract}

\section{Introduction}

In many countries, healthcare services are not currently provided in a sustainable manner. Healthcare systems and institutions are still largely based on providing acute and reactionary care; this approach is not well suited to the increasing burden of chronic disease in an aging population that requires more preventative and long-term care (World Health Organization, 2002: tinyurl.com/d5mf4dp). Thus far, the response to growing demand has been dramatic increases in health spending. Over the past 50 years, health spending has outstripped overall growth in GDP for OECD countries (OECD, 2011: tinyurl.com/bq4prbp). In Canada, health spending in the last decade has grown at double of the rate of revenue growth (CIHI, 2011: tinyurl.com/d86xla7).

However, as health spending reaches almost $40 \%$ of government budgets, the affordability of health has become a major economic and social issue (OECD, 2011: tinyurl.com/bq4prbp). Rising health spending is a concern not only for our ability to provide healthcare; it also represents a significant opportunity cost because there is less room in government budgets for spending and in- vestments in other areas such as education and the environment, which are increasingly being recognized as important determinants of health.

There is an urgent and dire need to innovate both the practice and delivery of healthcare. There is also a unique opportunity for social innovators to build enterprises with business models that can make a positive social impact by improving the sustainability of healthcare, which itself is a large and resilient market.

This article highlights the urgent need for innovation in the healthcare sector and the role that information technology can play. First, the article describes the failures of top-down approaches and the inadequacies of incomplete bottom-up approaches. Next, the potential of short-term, intense collaborations between developers - called "hackathons" - is described, focusing on a unique type of hackathon, called "Hacking Health", which brings together healthcare professionals and software developers to quickly create working prototypes of new applications. This approach to social innovation using information technology holds promise for improving the quality and sustainability of healthcare. 


\section{Hacking Health: Bottom-up Innovation for Healthcare}

\section{Jeeshan Chowdhury}

\section{Top-down Failures}

Based on past results, top-down approaches from either political or corporate leadership seem seems less and less likely to address problems with healthcare sustainability. Attempts to restrain public spending on healthcare and efforts for reform have been largely unviable politically (Hacker, 2004: tinyurl.com/7f2wx54). While there has been growing investment in healthcare information technologies, the implementation of these large-scale projects by both governments and corporations has been slow and marred by cost overruns, delays, and in some cases, scandal (Dunleavy et al., 2006: tinyurl.com/89zyb4e; OECD, 2000: tinyurl.com/84b26yy). For example, in Ontario, the issue of eHealth is still largely seen as a political liability. The otherwise-adept Google permanently shut down their foray into the sector, "Google Health" this past year (Lohr, 2011; tinyurl.com/4xz8a3m).

At the same time, healthcare as a sector has been largely resistant to reform and change by information technology (Barnett et al., 2011: tinyurl.com/7ero9b5; Boonstra and Broekhuis, 2010: tinyurl.com $/ 7 \mathrm{~b} 559 \mathrm{cg}$ ). While there has been dramatic and disruptive change to almost every other sector and industry over the past decades, healthcare remains rooted in antiquated practices and systems. For example, banking and airlines have made significant improvements in service delivery and efficiency using information technologies, particularly with web and mobile innovations (Bower, 2005: tinyurl.com/7nv872j). The delivery of healthcare, however, remains largely unchanged despite the advances and impact that these innovations have had in almost every other aspect of daily life (Masys, 2002: tinyurl.com/6ljzka3).

It is paradoxical that, although medicine has made remarkable progress in diagnosis and treatment, the practice and delivery of healthcare remains largely unchanged. For example, there has been considerable advancement in diagnostic technologies. In the past two decades, CT and MRI have become common procedures. Innovation progresses at a rapid pace with the introduction of PET scans and new procedures are continuously in development. However, over the same period, pagers and fax machines have largely become outdated yet they remain the mainstay means of communications within hospitals and between institutions. It is a still common for a physician, who has a pager in one pocket and the smartphone in another, to communicate the results of an MRI by fax. This sophistication within disciplines alongside antiquated systems between individual silos is endemic in healthcare.

\section{An Incomplete Bottom-up Approach}

The spectacular failure of both governments and corporations in this space has demonstrated that technological innovation alone has been unable to make an impact in the way we practice and deliver healthcare. However, at the same time, there is a growing ecosystem of entrepreneurs and startups in healthcare (Deloitte, 2012; tinyurl.com/6uz7opj). These entrepreneurs bring an innovative approach to technology development that was initially pioneered by internet and mobile startups. Epitomized by Mark Zuckerberg in the Facebook shareholder's prospect as the "Hacker Way" (2012; tinyurl.com/7rt5xjl), this approach focuses on the rapid and iterative development of small but scalable projects. "Hacking" rapidly builds small prototypes that are immediately tested and refined and built up into full-scale products or services. This is essentially the opposite approach to large-scale government or corporate initiatives that require significant capital and investment upfront to build a completed version that is then imposed on a system.

Nevertheless, these smaller initiatives have faced the same resistance to change by health institutions and professions that have bogged larger players including governments and established corporations. Thus, the majority of health startups remain relegated to fitness and wellness applications. Fitness and wellness are important dimensions of health and they represent large and lucrative markets. However, these applications fail to address the fundamental clinical or medical practices and systems that are in direst need of innovation (Maqubela, 2012; tinyurl.com/bo3xc4b).

The "Hacker Way" has been successful outside of health because the developer or hacker can identify themselves as the final user of the product or service. In other words, developers for the most part create products that they themselves would use. In travel, banking, media, and social networking the developers have easy access to the fundamental users: either themselves or the people around them. However, in health, there is barrier of access to users. Health professions are highly regulated and it is a significant investment to enter these fields. Thus, just as healthcare suffers from silos within the sector, healthcare itself is in a silo, separated from other fields and disciplines.

The barrier to useful clinical applications may likely be due the fact that in healthcare, unlike in other sectors, the developers of technology are not the end-users. The "scratch your own itch" approach breaks down. Simil- 


\title{
Hacking Health: Bottom-up Innovation for Healthcare
}

\author{
Jeeshan Chowdhury
}

arly, because of the load of both training and daily operations, it is difficult for highly trained clinicians to also be skilled proficiently in the technical skills that are required for technology development. This situation is evident from a survey of healthcare startups and the products and services that being offered - they are for the most part limited to those user experiences accessible to developers, such as applications for diabetes management, scheduling services to assist in booking appointments, researching of medical information, and products geared for fitness or weight loss.

\section{Hackathons}

The overall barrier to more web and mobile solutions in healthcare is not itself an issue of technical innovation but rather a social innovation. Fundamentally, those with the technical skills to build solutions are separated from those who have the frontline experience and understanding to know which solutions should be built. The need for multidisciplinary approaches to healthcare itself is not new. There is a plethora of academic and industry programs that aim to either train health professionals with outside skills or to bring expertise from outside sectors into healthcare. However, these programs are long-term investments that can, over time, engender a more integrative approach to healthcare from practitioners and administrators.

Alternatively, the "hacker way" is embodied in practice by hackathons: small events where, over the course of day or week, programmers and developers collaborate intensely to build prototypes. These marathon events focus on rapidly iterative software development through which groups design, code, and build testable prototypes. Hackathons are well established in software companies and the model has been applied to specific software applications including health.

Steven Levy first wrote of students working in "marathon bursts" at MIT in the 1960s in Hackers: Heroes of the Computer Revolution (tinyurl.com/c6j85ey). Since then, hackathons have evolved to become routine and integral to development in companies such as Facebook, Foursquare, and Yelp. Their appeal has spread beyond just technology companies; Wired magazine reported that, in 2011, over 200 hackathons were held in the US alone (Leckart, 2012; tinyurl.com/6nktvpm). As the barriers to distribute and market software have fallen, especially through mobile platforms, hackathons have moved from rapid research and development within firms to entrepreneurial generation of new products and companies.
The impact of hackathons on entrepreneurship and creating lasting businesses is difficult to measure. However, there is growing anecdotal evidence of companies forming from hackathons, including the oftencited example of Group.me, a mobile messaging app that was acquired by Skype. Startup Weekend, a hackathon styled event, self reports that $36 \%$ of groups that form at their events continue to work together after three months (startupweekend.org/about/). Perhaps the best indication of the value of hackathons is the growing interest from established firms and venture capitalists. Microsoft and Nokia have each hosted hackathons as a means to spur innovative use of their products (microsoft .com/techedonline/). Venture capitalists are increasingly attending and judging hackathons as means to quickly identify potential ideas and teams.

\section{Hacking Health}

With the urgent need for disruptive innovation in health and the social barriers to change that currently exist, a group of young professionals spanning medicine, technology, and policy proposed an experiment to test whether the hackathon model could be applied not only to the development of projects but also to interdisciplinary teams. The experiment was Hacking Health (hackinghealth.ca), the first health-focused hackathon in Canada. The short-term goal was to develop working software that can immediately improve healthcare by solving known, bite-sized problems. More importantly, the long-term goal was to nurture ongoing collaborative partnerships between technology innovators and healthcare experts.

Unlike other hackathons, Hacking Health's primary goal was not simply to spur the development of interesting projects, but it was to attempt to narrow the gap between frontline health professionals and technology experts in rapid but lasting way. It is necessary, but insufficient, to simply put different groups of people in the same room. The physical gap between the two groups should be seen more as a function of the social gap between them. Both health professionals and technology experts are highly trained in their own demanding domains, each with their own technical jargon and ingrained methods and perspectives. Thus at Hacking Health, there was considerable focus on educating and training each group to be able speak to one another. For example, before the actual event, we hosted a "pitch clinic" for health professionals to provide training and coaching on how to communicate effectively with technical talent. Similarly, for technical talent, we held a "meet up" days before the event to introduce 


\section{Hacking Health: Bottom-up Innovation for Healthcare}

\section{Jeeshan Chowdhury}

software developers and web designers to the unique challenges of building solutions for healthcare.

Further, the open and rapid nature of hackathons is in stark contrast to the often regulated and incremental progress of clinical medicine. A number of the clinicians initially expressed concern of losing control of an important idea or ownership of intellectual property in such a setting. Fortunately, even if there is sensitive information, the short format of the hackathon is ideal for testing out one part of a larger vision. By analogy, one can still put together most of a recipe but hold back on sharing the special ingredients behind that secret sauce. Ultimately, for clinicians that may have valuable intellectual property, the real value will never be actualized unless they can execute. Even if a hacker could build their idea, they cannot test and implement it without a clinical partner. What assuaged clinicians is that both parties, health professional and hacker, need each other to move from idea to working product. At the level of the hackathon, intellectual property is owned by the team and it is up to individual teams to determine how it is shared. That being said, considering the short nature of the hackathon and very early nature of any prototypes formed, very little actual value resides in the limited code or early concepts produced at the hackathon itself. Ultimately, the true value lies in the team that has formed and the evolution of the members and project over time. If needed, the team may start afresh from the original code and concept worked on at the hackathon itself.

The purpose of a hackathons can be mainly a social or educational event, or it may be focused directly on launching apps; from the onset, Hacking Health was designed to catalyze entrepreneurial teams and projects to address issues in healthcare through business models. While a small number of teams pursued projects that were nonprofit, the social business model encouraged to the majority of teams was well received by both clinicians and technical experts. The clinicians who had identified problems that could be improved by technology and made the effort to attend Hacking Health also tended to be not only early adopters of technology but entrepreneurial as well. In interviews with developers and designers, many cited personal frustrations with the healthcare system as a primary motivator in attending and described a strong desire to use their skills to address those frustrations and access a robust and growing vertical market.

The first Hacking Health held in Montreal attracted over 200 health professionals and technical talent who produced 19 working prototypes over the course of the two-day event. Projects ranged from a prescription drug reminder application for patients, to a 3D burnarea calculator for physicians, which uses the off-theshelf Kinect video game accessory. The results can be seen at projects.hackinghealth.ca and this model will be replicated in similar events across Canada.

The first Hacking Health event demonstrated that interdisciplinary teams can be formed in a short period of time at a very low cost. However, it remains to be seen how this level of activity can be sustained over the medium and long term to the final goal of startup companies that are making a profitable and positive impact on healthcare. The next phase is supporting the self-selecting individuals that form nascent teams along the funnel of development. This will require a different set of incentives and supports apart from those that bring the teams together in the first place. This can be practically achieved by connecting the teams that form at Hacking Health to the growing ecosystem of startup incubators and accelerators that support early-stage entrepreneurs.

\section{Conclusions}

Information technologies, namely web and mobile services, have significant impact on a number of industries and sectors. These technologies are thus a keen target for social innovators to bring about change in their respective domain. While technology is always advancing and costs over time will reduce, the primary barrier to the introduction of these technologies to health, education, and other social sectors is no longer technical but rather a social question of how to best implement these solutions into a unique context.

The experiment of Hacking Health has shown that the rapid iterative mindset of hackers and hackathons is applicable not only to the development of prototypes but also teams that have a truly interdisciplinary approach to overcome social barriers to innovation. The key is to break down the barriers between technical experts who can build innovative technologies and the frontline practitioners who know which solutions can make an impact. Overcoming these barriers begins physically by placing both groups together in the same space but extends into bridging the language and cultural gaps between them.

The model of Hacking Health will be expanded to other cities starting first with Toronto in the fall of 2012. While a hackathon catalyzes early action and connec- 


\section{Hacking Health: Bottom-up Innovation for Healthcare}

\section{Jeeshan Chowdhury}

tions, to form lasting start-up enterprises, these early teams and ideas will require nurturing and support to progress through the innovation pipeline. As such, in the future Hacking Health will begin to investigate and develop mechanisms to translate the spurt of activity during the short period of the hackathon into long-acting startups.

The lack of empirical data on the long-term impact of hackathons in generating lasting entrepreneurial activity is an important area for future research investigation in this field. Designing support systems for teams after hackathons that also track their progress is an attractive means to both increase the throughput of teams through the innovation pipeline but also produce empirical evidence. Nevertheless, the low cost and risk of these types of events - where technology innovators are introduced and trained to work with frontline practitioners - makes the hackathon approach an accessible and scalable means to foster innovation in almost any social sector.

\section{About the Author}

Jeeshan Chowdhury is completing an MD/DPhil at the Universities of Alberta and Oxford, where he studied as a Rhodes Scholar. Jeeshan's academic research focuses on emerging healthcare technologies, namely developing novel point-of-care diagnostics and quantitative methods to measure the impact of health information systems. Through his academic experience, Jeeshan has co-founded HackingHealth.ca to foster health technology innovations. He also launched EnlightHealth.com, a health tech startup that innovates web and mobile solutions for patients, providers, and clinics.

Citation: Chowdhury, J. 2012. Hacking Health: Bottom-up Innovation for Healthcare. Technology Innovation (cc) BY Management Review. July 2012: 31-35. 